\title{
NEONATAL INTENSIVE CARE UNIT STUDY
}

\section{REPORT OF THE FIRST COHORT (1986-87) OF INFANTS}

T he aims of the National Intensive Care Unit Study were to establish a data base for specific groups of high-risk infants who were admitted to a Level 3 neonatal intensive care unit during the neonatal period, and to determine their survival and major disability rates.

The study population included all babies with a birthweight of 1000-5000 grams, who received at least four hours' mechanical ventilation which began in the neonatal period, and all babies with a birthweight of 400-999 grams (extremely low birthweight), who were admitted to a Level 3 neonatal intensive care unit (NICU) in NSW between July 1, 1986 and December 31, 1987.

At the start of the study, there were seven Level 3 NICUs in NSW. An eighth unit was opened recently at Nepean Hospital in Sydney's west. Two of the original seven units are in children's hospitals and the others are in Level 6 obstetric hospitals. Six of these Level 3 NICUs are within the Sydney metropolitan Health Areas and the seventh is in a Level 6 obstetric hospital in the Hunter Area.

Information on extremely low birthweight (ELBW) infants born in Level 1-4 obstetric hospitals was obtained from the NSW Maternal and Perinatal Data Collection, death certificates, birth registrations and emergency newborn transport records. For ELBW infants born in Level 6 obstetric hospitals, information was also obtained from the labour ward birth registers. Other sources of data were the admission books and hospital medical records.

Maternal and perinatal data for infants born from January 1 to December 31,1987 were obtained by manually matching the mothers' NSW Maternal and Perinatal Data Collection forms with the infants' NICU study forms.

Survival in relation to transfer status, level of hospital of birth, gender, plurality, gestational age, birthweight and congenital anomalies was reported at one week, four weeks and one year of age.

Follow-up assessment of ELBW infants, to document long-term survival and incidence of major disability at one and three years of age, corrected for prematurity, was arranged by the Level 3 NICU responsible for their neonatal care. Detailed physical, neurological, psychometric, ophthalmological and audiological examinations were performed by the relevant specialist. The National Acoustic Laboratory or equivalent audiology service assessed the hearing of 78 of 151 children ( 52 per cent).

Mechanically ventilated infants with birthweights 1000-5000 grams were followed up only in relation to survival to one year of age.

\section{RESULTS \\ 1. EXTREMELY LOW BIRTHWEIGHT INFANTS 400-999 GRAMS}

A total of 776 infants with birthweights $400-999$ grams were born to women resident in NSW during the study period. Of these, 298 ( 38 per cent) were stillborn, and 478 (62 per cent) were liveborn. Of the liveborn infants, 272 (57 per cent) were admitted to a Level 3 NICU. Sixty-five of the 776 infants ( 8 per cent) had a life-threatening congenital anomaly; 33 of those infants were stillborn, 28 died during the neonatal period, one died during the postneonatal period and three survived to one year. Slightly more male than female infants were born ( 399 or 51 per cent, compared with 377 or 49 per cent), and 646 of the infants ( 83 per cent) were singletons.

\section{Survival to one year of age}

Two hundred and sixty infants without life-threatening congenital anomalies were born alive in a Level 6 obstetric hospital. Of these, 211 ( 81 per cent) were admitted to a Level 3 NICU, and 131 (50 per cent) survived to one year, corrected for prematurity. Of the 13 born with a life-threatening congenital anomaly in a Level 6 obstetric hospital and admitted to a Level 3 NICU, only three survived.

In contrast, 47 ( 25 per cent) of the 186 infants without life-threatening congenital anomalies born alive in Level 1-4 obstetric hospitals were transferred to one of the seven Level 3 NICUs in NSW, and 17 ( 9 per cent) survived. One infant born with a life-threatening congenital anomaly who was transferred subsequently died.

All the infants born in a Level $1-4$ obstetric hospital and not transferred to a Level 3 NICU died.

Thirty-six per cent (148 of 413) of the liveborn ELBW infants without a life-threatening congenital anomaly survived to one year. However, the one-year survival rate of all ELBW infants without life-threatening congenital anomalies admitted to a Level 3 NICU in NSW was lower for those who were born in a Level 1.4 obstetric hospital and transferred ex-utero to the NICU ( 17 of 47 , or 36 per cent), than for those born in a Level 6 obstetric hospital and admitted to the NICU (131 of 211 , or 62 per cent) $(\mathrm{P}<0.01)$.

Infants without congenital anomalies were three times less likely to die when born in a Level 6 obstetric hospital, if their five-minute Apgar score was seven or more, or if they were singletons. Females were twice as likely to survive as males.

Major disability

Of the 151 children who reached a corrected age of one year, 13 ( 9 per cent) were lost to follow-up, 11 ( 7 per cent) were assessed at less than 10 months of age (corrected for prematurity), 109 ( 72 per cent) were assessed between 10 and 14 months, and 18 (12 per cent) were assessed at 15 months or older. 


\section{Neonatal intensive care unit study}

\section{Continued from page 3}

One hundred and twenty-seven children (84 per cent) have had a full neurological assessment at 10 months or older, but only 82 ( 54 per cent) have had both a full neurological and a standardised psychometric assessment. Twenty-one ( 16.5 per cent of 127$)$ of the children examined had one or more major disabilities, 9 ( 7 per cent) having cerebral palsy, 3 ( 2 per cent) being blind and 2 ( 1.6 per cent) deaf. Seven ( 8.5 per cent of 82) children had an isolated mental development delay on psychometric assessment while two children had cerebral palsy and mental developmental delay.

\section{MECHANICALLY VENTILATED INFANTS WEIGHING 1000-5000 GRAMS AT BIRTH}

Reasons for mechanical ventilation in this group of infants included respiratory failure, congenital anomalies, post surgery, perinatal asphyxia and sepsis. Of the 1293 infants, 318 ( 25 per cent) had congenital anomalies and they were analysed separately in relation to survival.

Overall, 89 per cent of the 975 infants without congenital anomalies survived to one year of age, with neonatal and postneonatal mortality rates of 10 and 1 per cent respectively.

The most important finding of the study was that a curvilinear relationship existed between birthweight and survival to one year of age with a peak in survival to one year of age (96 per cent) in infants with birthweights in the 2000-2499 gram range. Infants weighing 3000-3499 grams, 1750-1999 grams and 2000-2499 grams were respectively three, four and six times more likely to survive to one year than those weighing 3500-3999 grams. A similar relationship existed between gestational age and survival, with a peak ( 97 per cent) in survival for infants born at 33-34 weeks gestation. Infants of $35-36$ weeks gestation were three times, and those of 33-34 weeks six times, more likely to survive to one year than those of $37-41$ weeks gestation. Sixty-three per cent (202 of 318) of infants with congenital anomalies survived.

The aims of the Neonatal Intensive Care Unit Study data collection are being redefined: the study will audit patterns of usage of NICUs, patterns of diseases and mortality rates in NICUs, morbidity of infants at one, three, five and seven years of age, and the relationship of all these to maternal, perinatal and neonatal factors. The audit will serve as a quality assurance of neonatal resuscitation and transport, management of the infants in the NICUs and the follow-up assessment of these babies. It will also provide a comparison with the population-based data collected on all births in NSW.

Lee Sutton, Clinical Epidemiologist, NSW Neonatal Intensive Care Unit Audit.

Barbara Bajuk, Clinical Nurse Consultant/Coordinator, NSW Neonatal Intensive Care Unit Audit.

\section{Public health ABstractS}

D rofessor James S. Lawson, Head of the School of Health Services Management at the University of NSW, has prepared the following public health items from the literature.

\section{PROSTATE PROBLEM NUMBERS HIGH}

B enign hypertrophy of the prostate is a common reason for surgery in elderly men, but the incidence of this condition has not been known. A British-based study is of general interest as it has used modern techniques (rectal ultrasound) to asses the volume of prostates in more then 700 men between the ages of 40 and 79 years. The study found significant hypertrophy of the prostate in 138/1000 men aged 40 to 79 years rising to $430 / 1000$ men aged 60 to 69 years. This shows the condition much more common than was previously thought to be the case.

Garraway WM, Collins GN and Lee RJ. High prevalence of benign prostatic hypertrophy in the community. Lancet 1991; 338:469-471.

\section{GOOD NEWS ON ALCOHOL AND HEART DISEASE}

I he association between alcohol consumption and reduced risk of coronary heart diseảse has been well documented. However, despite these studies, some have argued that the association may be due, in part, to the use in studies of non-drinkers who may include heavy drinkers who have stopped drinking because of illness. A very large American study involving more than 50,000 male health professionals has been conducted to determine whether the relationship between alcohol and heart disease is confirmed.

After adjustment for the standard coronary heart disease risk factors, the study has shown it is probable that alcohol consumption does reduce heart disease. It should be noted that the alcohol consumption by the study group was relatively light and it should not be assumed that heavy drinking will reduce heart disease risk further.

Rimm EB, Giovannucci EL, Willett WC, Colditz GA et al. Prospective study of alcohol consumption and risk of coronary disease in men. Lancet $1991 ; 338: 464-468$.

\section{THE VALUE OF METHADONE}

$\mathrm{n}$ the quarter-century since methadone was introduced as a treatment for heroin addiction, its effectiveness has been demonstrated throughout the world. Methadone has been found to be an attraction to a significant proportion of the addict population on a voluntary basis, with high retention rates and high cessation of heroin use.

Opposition to methadone programs almost universally attracts controversy and difficulty. Robert Newman, one of the key founders of methadone projects, has written about this rejection phenomenon. He has concluded that, in part, the problem is due to the widely-held perception that heroin addicts are incorrigible hedonists and therefore raise a good deal of intolerance in the community.

Newman RG. What's so special about methadone maintenance? Drug Alcohol Rev 1991; 10:225-232. 